\title{
Neural Network-Based Matrix Completion for Minimal Configuration of Body Surface Potential Mapping
}

\author{
Darek Bizcaino ${ }^{1}$, Kamil Bujnarowski ${ }^{1}$, Maksymilian Matyschik ${ }^{1}$, Henry Mauranen ${ }^{1}$, Ruhui Zhao ${ }^{1}$, \\ Pietro Bonizzi ${ }^{1}$, Joël Karel ${ }^{1}$ \\ ${ }^{1}$ Department of Data Science and Knowledge Engineering, Maastricht, The Netherlands
}

\begin{abstract}
Body surface potential mapping (BSPM) provides high spatial resolution recordings of the electric potential of the heart on the body surface. BSPM can involve up to 200 electrodes, in contrast to standard 12-lead ECG. The costs and complexity of a BSPM procedure are a limiting factor to its use in clinical practice. Both can be reduced by using fewer electrodes and reconstructing signals from the missing electrodes with an artificial neural network. The minimal configuration consists of the electrodes that are most relevant for reliable reconstruction. We propose an architecture for a variational autoencoder, trained on BSPM procedures from the Nijmegen dataset: EDGAR [1], to reconstruct a full 65-lead system from a reduced number of input electrodes. Further, we determine the effect of an increased numbers of missing electrodes on the corresponding reconstruction error, and show that it is possible to achieve a good 65-lead reconstruction from as few as 12 electrodes. We consider the implication of our research in the scope of current BSPM practice, as well as the limitations of using neural networks for this task.
\end{abstract}

\section{Introduction}

Heart diseases are among the most common causes of death worldwide [2]. Today, the 12-lead electrocardiographic (ECG) system remains a standard cardiovascular diagnostic procedures among medical professionals. However, the accuracy of the 12-lead ECG has been called into question due to the fact that necessary diagnostic information may not be captured by this recording system, for instance when detecting acute myocardial infarction (AMI) [3]. Other studies have shown that by using Body Surface Potential Map (BSPM) recordings, a higher degree of accuracy is gained in correctly detecting heart conditions [3]. BSPM also measures the electrical potential of the heart on the body surface, but it employs (many) more electrodes then the standard 12-lead ECG [4]. The additional information gained with BSPM is used, among all, when finding a reliable solution to the inverse problem in electrocardiography (also known as ECG-Imaging). Although superior in terms of diagnostic yield, BSPM is not widely used in clinical practice. This may be due to the higher costs associated with this procedure, more time and trained personnel needed for the set up, and the lack of a standardized BSPM measurement system. More than 11 BSPM systems [5] could be found during our literature review, which have completely different electrode counts (from 32 to 219) and electrode positions.

One way to overcome these limitations would be to use partial data from a limited (and optimal) set of body surface electrodes in order to reconstruct the whole BSPM data matrix [6]. Several studies have addressed the question of how many electrodes are necessary with the purpose of completing partial BSPM data in the study of atrial fibrillation (AF). In [7], researchers argued that it has been generally accepted that 30-32 electrodes are sufficient. In [4], it has been proven that 23 electrodes are needed to reconstruct the AF data with $25 \%$ error rate, while 17 and 8 electrodes are required for rebuilding $\mathrm{QRS}$ complex and $\mathrm{P}$ wave, respectively.

This study proposes the use of an artificial neural network to reconstruct missing BSPM recordings starting from a limited set of BSPM electrodes. The reconstruction of the data from the missing electrodes is achieved by using a variational autoencoder (VAE). First the optimal architecture for the VAE and the corresponding optimal parameter setting are investigated. Next, the minimal BSPM electrode configuration required for an accurate reconstruction is determined, and qualitatively compared with the standard 12-lead ECG.

\section{Methodology}

\subsection{Data}

The data used in this study were retrieved from the EDGAR Time Signal Catalog, contributed by the Radboud University in Nijmegen, Netherlands [1]. The dataset consisted of BSMP measurements from a single healthy 


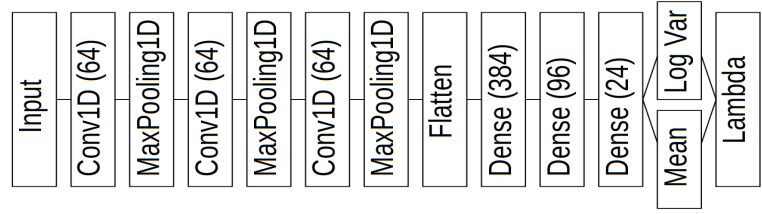

Figure 1. Structure of KurtNet's encoder. Decoder is a mirrored structure from last dense layer to the first convolutional one.

male patient ( 65 electrodes 12 on the back and 53 on the front). Recordings were taken over 13 different procedures. Recordings from one procedure were used to train the network, while all the other recordings were used for validation and for determining the minimal BSPM configuration. The signals were downsampled to $512 \mathrm{~Hz}$ from $1024 \mathrm{~Hz}$. Breathing frequencies were removed from the signals with a high-pass Chebyshev II filter of order 2 and cutoff of $0.5 \mathrm{~Hz}$.

\subsection{VAE architecture}

We considered three different architectures for the VAE: image classification networks AlexNet [8] and VGGNet [9], and KurtNet, which is based on an autoencoder design generated in house. Figure 1 shows the encoding part of KurtNet, which has significantly fewer parameters due to the smaller number of units in each convolutional and dense layer. In order to transform these networks into appropriate VAEs, they had to first be expanded after their output layer, which was repurposed as a sampling layer. The encoder - decoder architecture was obtained by mirroring the previous layers and exchanging downsampling layers for upsampling layers. Other than the input layer and the sampling layer, the network architectures were fixed. This way, it was possible to keep the amount of hyperparameters under control. The input layer corresponds to all samples from all BSPM leads in a given time interval (window), while the sampling layer is considered the network's internal representation of the data. All three architectures were tested with sampling (or latent) layer dimension of 12, 18, 24 nodes and a window length of 2, 3 and 4 seconds. Performance was assessed in terms of mean squared reconstruction error between the reconstructed and the original signals.

\subsection{Minimal BSPM configuration}

In order to find the most significant electrodes for the reconstruction, we systematically attempted to reconstruct the full BSPM data matrix after excluding an iteratively increasing number of electrodes from the input. Missing recordings were replaced with zeros. This can distort the features learned from the measurements, and we counteracted it by reiterating the reconstruction $n$ times (by reapplying the forward pass through the network while replacing the missing recordings with their reconstructions). Several values of $n$ were tested, to investigate its influence on the reconstruction error.

Testing all possible electrode combinations would have been far too computationally intensive, as for our dataset there are 65 ! possible electrode combinations. Therefore, we decided to iteratively drop the least significant electrodes, until no electrode was left. All corresponding configurations were tested and the set of 12 electrodes that gave the lowest reconstruction error was selected as the minimal BSPM configuration for this study. The locations of the 12 most important electrodes were then visually compared to the standard 12-lead ECG.

\section{Results}

\subsection{VAE architecture}

Figure 2-left shows the evolution of the validation error of the three architectures over the training epochs (with all networks trained for 50 epochs). Performance of the three networks was tested for different window lengths (of 2, 3, and 4 seconds), and latent layer sizes (of 12, 18, and 24). Figure 2-right shows the final validation errors for KurtNet and AlexNet, for all possible combinations of the window length and latent layer size. The best architecture chosen for further experiments was KurtNet with a window length of 2 seconds and a latent layer size of 24 (KW2L24).

\subsection{Minimal BSPM configuration}

Figure 3 shows the reconstruction error as a function of the number of less significant electrodes dropped and the number $n$ of reconstruction iterations in the network. Figure 4 shows a reconstruction of 64 BSMP recordings from the 12 most significant electrodes (for signals the network was not trained on), marked with green boxes. $n=12$ was used to achieve this reconstruction, as it was associated with a robust reconstruction over different electrode drop counts (based on the results showed in Figure 2). When performing such reconstruction with 12 electrodes, we obtained a mean square error of $11.210 \pm 0.408 \mathrm{mV}$ over 100 predictions. Finally, Figure 5 shows the BSPM system mapped to the human torso with the final 12 most relevant leads shown in red.

\subsection{Conclusions}

In this study, we presented an approach for the reconstruction of signals from missing BSPM electrodes based on a variational autoecoder. We evaluated three possible 

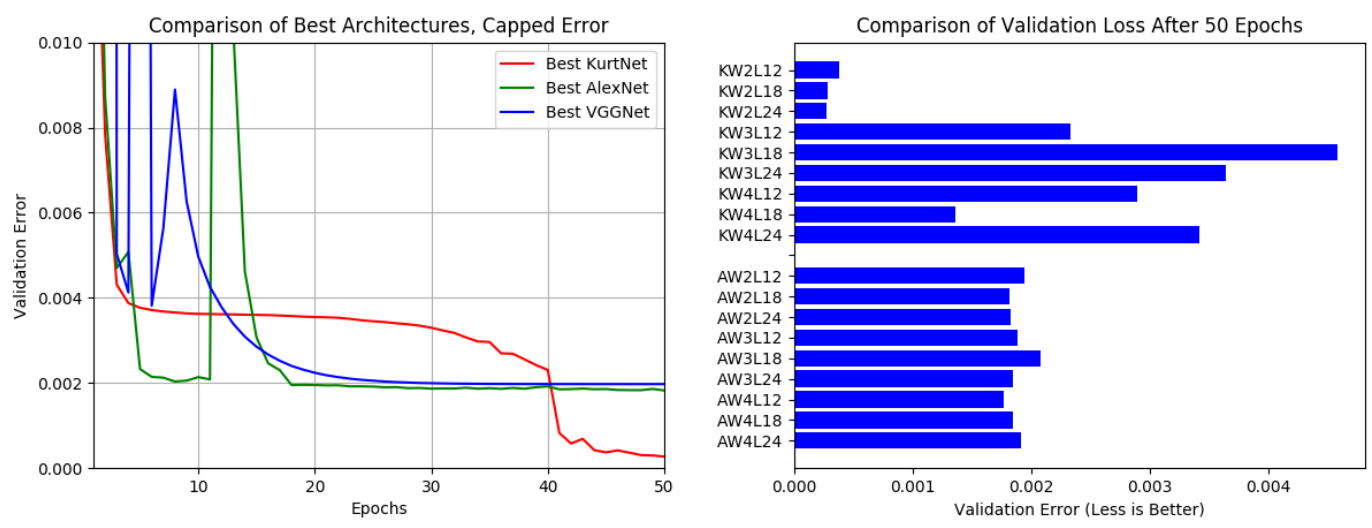

Figure 2. (Left) Validation error over 50 training epochs for KurtNet (red), AlexNet (green) and VGGNet (blue). (Right) Final validation error after training for 50 epochs for KurtNet and AlexNet to show the different effects of hyperparameters: window sizes of 2, 3, 4 seconds and latent dimensions 12, 18, 24 .

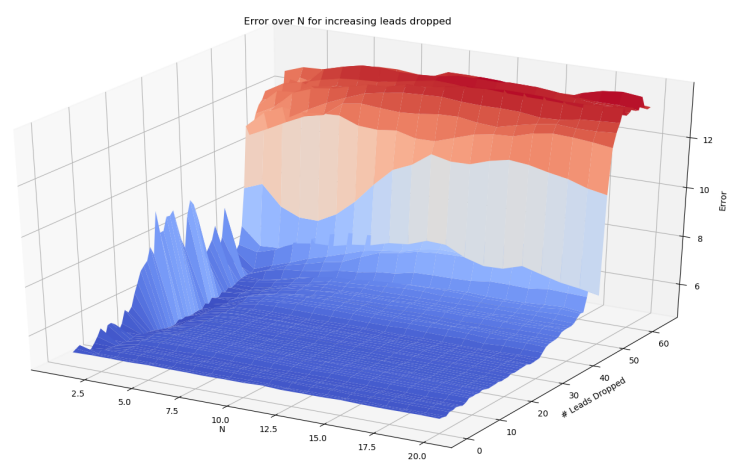

Figure 3. Reconstruction errors using KW2L24. The color gradient shows dark blue for low values and red for high values. $\mathrm{X}$ axis shows the number of reconstructions $n, \mathrm{Y}$ axis is the number of leads dropped and $\mathrm{Z}$ axis shows the corresponding error. The main area of interest it the dark blue plateau of low error values before the error climbs as too many electrodes are missing.

architecture for the autoencoder, AlexNet [8], VGGNet [9], and KurtNet, which was developed in house. KurtNet showed the best performance on the validation data set, and it was able to capture the features of the ECG signals better than the former two (which sometimes predicted simple straight lines).

When looking for the minimum BSPM configuration, we noticed that dropping up to about 25 electrodes does not have a strong impact on the reconstruction error. However, when over half of the electrodes are missing, it is necessary to repeat the forward pass up to 8 times to have a reliable reconstruction. When most of the electrodes are missing, it no longer matters how many reconstruction passes are performed, as there is too much missing information, and the additional reconstructions are detrimental as they will reinforce any erroneous patterns in the wave-forms. In this study, we could achieve a good reconstruction with just 12 electrodes. From Figure 4 it can be seen that there is still a visible difference between the reconstructed and the original signals, but the positions of peaks and general waveform morphologies are well modelled by the neural network. We also observed that sometimes out of two peaks present in the ground truth signal, only one was reconstructed properly. We assume this may be caused by the model being over-fitted to the training data and the features of the peaks not being prevalent enough to be captured by the autoencoder. Repetition through the network then naturally increases this error, reconstructing only a single peak. This will need to be investigated in a future study. When considering the positions of the most relevant electrodes for reconstruction onto the human torso, we could notice a good agreement between those and the locations of the precordial leads in the standard 12-lead ECG (left chest and lower-left back areas in Figure 5). Moreover, we could notice the appearance of few more leads on the back. This suggests that adding a few extra electrodes to the standard 12-lead ECG may be enough to provide an accurate reconstruction of BSPM recordings by means of ANN.

\section{References}

[1] Aras K, Good W, Tate J, Burton B, Brooks D, Doessel O, Schulze W, Patyogaylo D, Wang L, Van Dam P, MacLeod R, Coll-Font J. Experimental Data and Geometric Analysis Repository: EDGAR. Journal of Electrocardiology 2015; 48(6):975-981.

[2] Pagidipati N, Gaziano T. Estimating deaths from cardiovascular disease: a review of global methodologies of mortality measurement. Circulation 2013;127(6):749-756.

[3] Ornato J, Menown I, Peberdy M, Kontos M, Riddell J, May- 


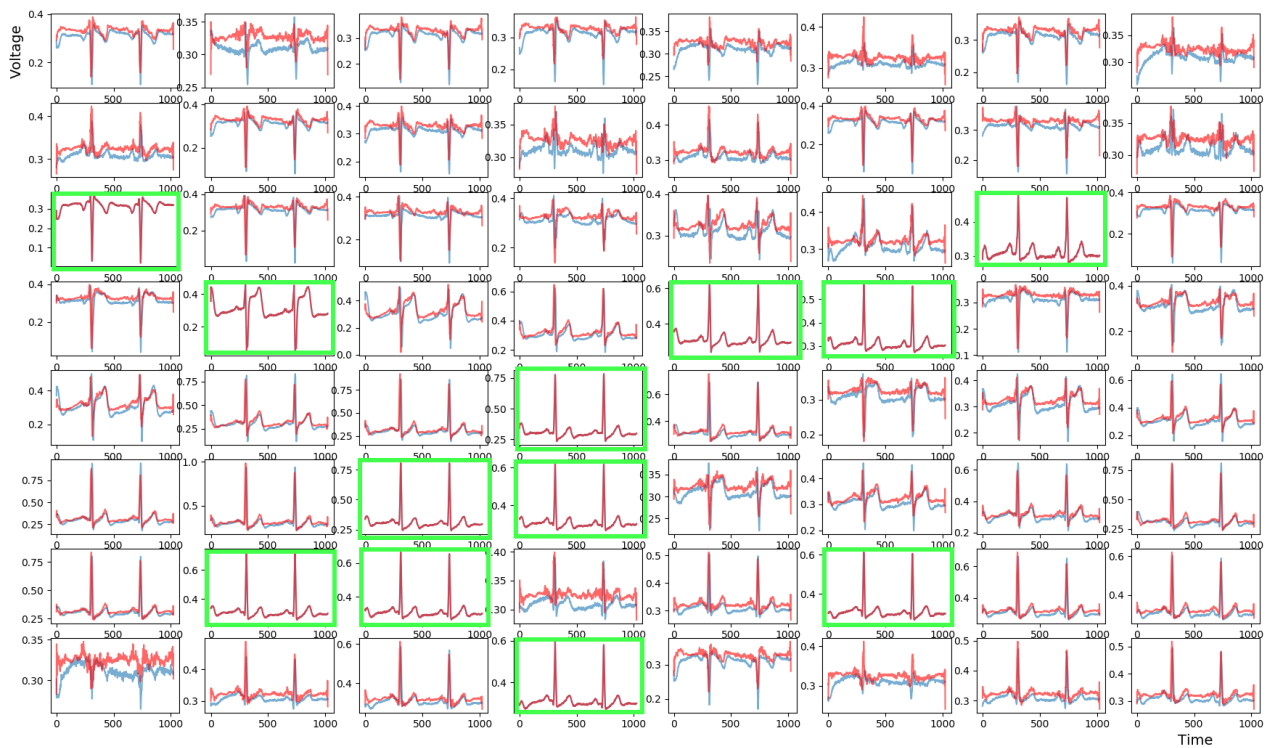

Figure 4. Ground Truth signal (blue) and KW2L24 reconstruction (red) for data the network was not trained on after $n=12$. Reconstruction using 12 best leads marked by green frames.
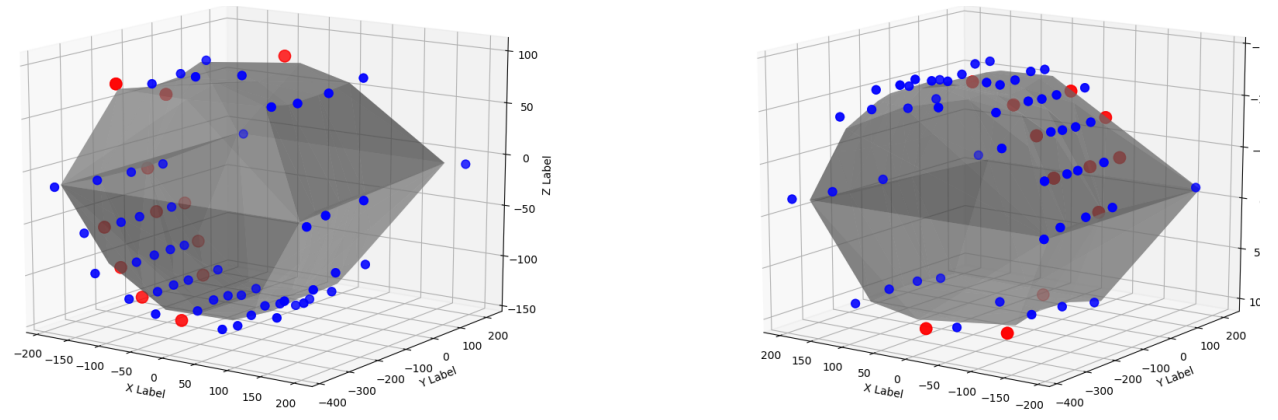

Figure 5. Representation of 12 most significant leads from Figure 4. Patient lying with head into the paper facing down (left) and up (right).

nard S, Higgins G, Adgey J. Body surface mapping vs 12lead electrocardiography to detect ST-elevation myocardial infarction. American Journal of Emergency Medicine 2009; 27(1):779-784.

[4] de la Salud Guillem M, Bollmann A, Climent A, Husser D, Millet-Roig J, Castells F. How many leads are necessary for a reliable reconstruction of surface potentials during atrial fibrillation? IEEE Transactions on Information Technology in Biomedicine 2009;13(3):330-340.

[5] Hoekema R, Uijen G, Van Oosterom A. On selecting a body surface mapping procedure. Journal of Electrocardiology 1999;32(2):93-101.

[6] Finlay D, Nugent C, Wang H, Zheng H, Donnelly M, McCullagh P. Classification of Body Surface Potential Maps: A Comparison of Isointegral Measurements in the Diagnosis of Old Myocardial Infarction. European Journal for Biomedical Informatics 2007;3(1):en7-en12.

[7] Kligfield P, Gettes L, Bailey J, Childers R, Deal B, Hancock
W, Van Herpen G, Kors J, Macfarlane P, Mirvis D, Pahlm O, Rautaharju P, Wagner G. Recommendations for the standardization and interpretation of the electrocardiogram. Circulation 2007;115(10):1306-1324.

[8] Krizhevsky A, Sutskever I, Hinton GE. ImageNet Classification with Deep Convolutional Neural Networks. In Proceedings of the 25th International Conference on Neural Information Processing Systems - Volume 1, NIPS'12. USA: Curran Associates Inc., 2012; 1097-1105.

[9] Simonyan K, Zisserman A. Very Deep Convolutional Networks for Large-Scale Image Recognition. arXiv 2015;

Address for correspondence:

Joël Karel

P.O. Box 616, 6200 MD, Maastricht, The Netherlands joel.karel@maastrichtuniversity.nl 